\title{
The Dark Matter Halo Density Profile, Spiral Arm Morphology, and Supermassive Black Hole Mass of M33
}

\author{
Marc S. Seigar ${ }^{1,2}$ \\ ${ }^{1}$ Department of Physics and Astronomy, University of Arkansas at Little Rock, 2801 S. University Avenue, Little Rock, \\ AR 72204-1099, USA \\ ${ }^{2}$ Arkansas Center for Space and Planetary Sciences, 202 Old Museum Building, University of Arkansas, Fayetteville, \\ AR 72701, USA \\ Correspondence should be addressed to Marc S. Seigar, mxseigar@ualr.edu
}

Received 28 January 2011; Accepted 3 March 2011

Academic Editor: N. Caon

Copyright () 2011 Marc S. Seigar. This is an open access article distributed under the Creative Commons Attribution License, which permits unrestricted use, distribution, and reproduction in any medium, provided the original work is properly cited.

We investigate the dark matter halo density profile of M33. We find that the HI rotation curve of M33 is best described by an NFW dark matter halo density profile model, with a halo concentration of $c_{\mathrm{vir}}=4.0 \pm 1.0$ and a virial mass of $M_{\mathrm{vir}}=(2.2 \pm 0.1) \times 10^{11} \mathrm{M}_{\odot}$. We go on to use the NFW concentration $\left(c_{\mathrm{vir}}\right)$ of M33, along with the values derived for other galaxies (as found in the literature), to show that $c_{\text {vir }}$ correlates with both spiral arm pitch angle and supermassive black hole mass.

\section{Introduction}

The currently favored cosmological model, lambda+ cold dark matter $(\Lambda \mathrm{CDM})$, is remarkably successful at reproducing the large-scale structure of the Universe $[1,2]$. However, small-scale observations have proven harder to explain. High-resolution N-body simulations of $\Lambda \mathrm{CDM}$ structure formation predict that the central density profiles of dark matter halos should rise steeply at small radii, $\rho(r) \propto r^{-\gamma}$ with $\gamma \simeq 1-1.5$ ([3], henceforth NFW, $\left.[4,5]\right)$. Observations of rotation curves of late-type disk galaxies and dwarf galaxies, on the other hand, have shown that quite often, mass distributions with lower than predicted densities or with constant density cores, where $\gamma \simeq 0$ (i.e., a pseudoisothermal profile), are preferred [6-13]. This is known as the cusp/core problem. One possibility is that these observations are pointing to a real problem with $\Lambda \mathrm{CDM}$ cosmology, perhaps indicating that the dark matter is not cold but rather warm [14], in which case, it is easier to produce constant density cores at the centers of dark matter halos. Another possibility is that these late-type galaxies have constant density cores because of their late formation [15] and that earlier-type bulge-dominated galaxies (which form at earlier times) will tend to conform to the standard expectations of the theory. This is because the central mass densities of galaxies tend to reflect the density of the Universe at their formation time [15].

In this paper, we have chosen to model the HI rotation curve of M33 from Crobelli and Salucci [16]. Due to its proximity, M33 can be studied in exquisite detail, and it, therefore, provides a crucial testing ground of our ideas of galaxy formation. Its Hubble classification is SA(s)cd [17], meaning that it is of particularly late type, with little or no bulge. This is reflected in the central supermassive black hole mass of $M_{\mathrm{BH}}<1500 \mathrm{M}_{\odot}$ [18], and black hole masses tend to be related to the central bulge mass $[19,20]$. In this paper we model the rotation curve of M33 with both a pseudoisothermal profile dark matter halo density model and an NFW dark matter halo density model. We then use parameters derived from these fits to look at relations between the dark matter halo and other galaxy properties, such as supermassive black hole mass and spiral arm pitch angle.

This paper is organized as follows. Section 2 describes the observed data and data analysis. Section 3 describes how the rotation curve is modeled and how we derive the baryonic and dark matter halo contributions to the rotation curve. Section 4 discusses our results, and Section 5 summarizes our findings. Throughout this paper, we assume a flat $\Lambda \mathrm{CDM}$ cosmology with $\Omega_{m}=0.27$ and a Hubble constant $H_{0}=$ $75 \mathrm{~km} \mathrm{~s}^{-1} \mathrm{Mpc}^{-1}$. 


\section{Observations and Data Reduction}

We have made use of the Spitzer/IRAC 3.6- $\mu \mathrm{m}$ image of M33. The IRAC observations were taken as part of the Gehrz Guaranteed Time Observer Program ID 5. The mapping sequence for each epoch consisted of $\simeq 148$ positions per channel. Each position was observed with three $12 \mathrm{~s}$ frames dithered with the standard, small, cycling pattern. The FWHM of the point-spread function (PSF) at 3.6- $\mu \mathrm{m}$ is $1.7^{\prime \prime}$ or $6.9 \mathrm{pc}$ at the distance of M33. The final mosaic spans an area of $\sim 1 .^{\prime} 0 \times 1 .^{\prime} 2$. We adopt a distance to M33 of $d=$ $840 \mathrm{kpc}$ (e.g., [21]), and it has a redshift of $z=-0.000597$ [17].

For dynamical measurements, we make use of the HI rotation curve of Corbelli and Salucci [16]. We also make use of the inclination corrected HI linewidth from HyperLeda (http://leda.univ-lyon1.fr/) of $100.4 \pm 3.0 \mathrm{~km} \mathrm{~s}^{-1}$ (e.g., [22]).

For the determination of the spiral arm morphology we have made use of an $R$ band image from the digital sky survey (DSS).

2.1. Measurement of Spiral Arm Pitch Angle. Spiral arm pitch angles are measured using a two-dimensional fast Fourier decomposition technique, which employs a program described in Schröder et al. [23]. Logarithmic spirals are assumed in the decomposition.

The amplitude of each Fourier component is given by

$$
A(m, p)=\frac{\sum_{i=1}^{I} \sum_{j=1}^{J} I_{i j}(\ln r, \theta) \exp -\left[i\left(m \theta_{p} \ln r\right)\right]}{\sum_{i=1}^{I} \sum_{j=1}^{J} I_{i j}(\ln r, \theta)},
$$

where $r$ and $\theta$ are polar coordinates, $I(\ln r, \theta)$ is the intensity at position $(\ln r, \theta), m$ represents the number of arms or modes, and $p$ is the variable associated with the pitch angle $P$, defined by $P=-\left(\mathrm{m} / p_{\max }\right)$. Throughout this work, we measure the pitch angle $P$ of the $m=2$ component.

Pitch angles are determined from peaks in the Fourier spectra, as this is the most powerful method to find periodicity in a distribution $[24,25]$.

The image was first projected to face on. Mean uncertainties of position angle and inclination as a function of inclination were discussed by Considère and Athanassoula [24]. For a galaxy with low inclination, there are clearly greater uncertainties in assigning both a position angle and an accurate inclination. These uncertainties are discussed by Block et al. [26] and Seigar et al. [27, 28] who took a galaxy with low inclination $\left(<30^{\circ}\right)$ and one with high inclination $\left(>60^{\circ}\right)$ and varied the inclination angle used in the correction to face on. They found that for the galaxy with low inclination, the measured pitch angle remained the same. M33 has a relatively low inclination of $\sim 30^{\circ}$, and so the uncertainty in the inclination angle in this case does not result in a large error in the pitch angle we measure for M33. Our deprojection method assumes that spiral galaxy disks are intrinsically circular in nature.

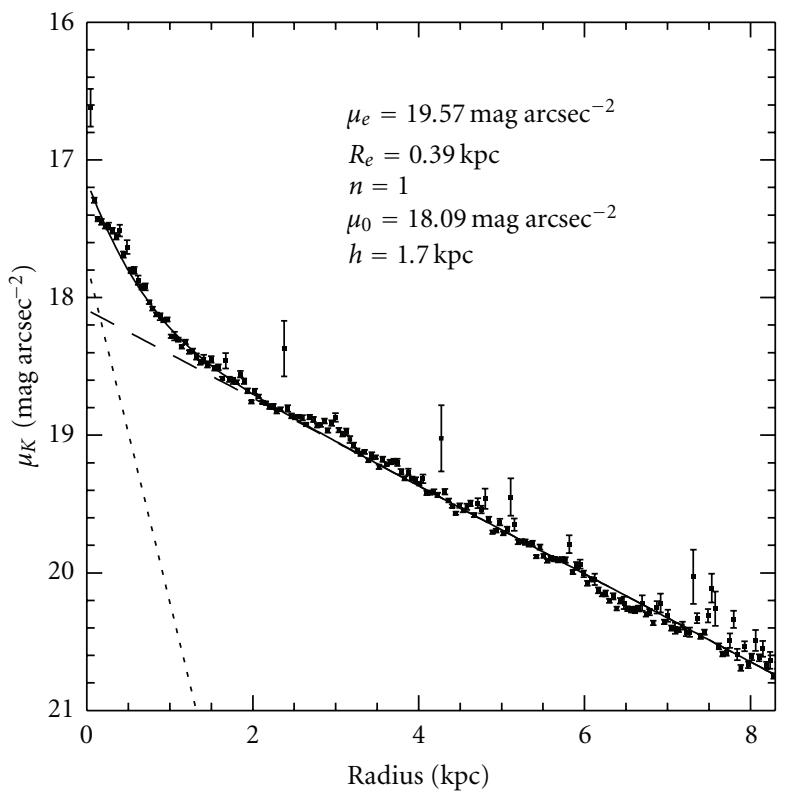

Figure 1: The Spitzer 3.6- $\mu \mathrm{m}$ surface brightness profile with decomposition into bulge and disk components. The bulge has been fitted with a Sérsic model (short-dashed line), and the disk has been fitted with an exponential model (long-dashed line).

\section{Mass Modeling}

3.1. The Baryonic Contribution. Our goal is to determine a mass model for M33 from the direct fitting of mass models to its rotation curve. We perform a bulge-disk decomposition in order to estimate the baryonic contribution. We then determine several different models and try to recreate the nuclear spiral by minimizing reduced- $\chi^{2}$.

We first extract the surface brightness of M33 using the Spitzer 3.6- $\mu \mathrm{m}$ image and the IRAF ELLIPSE routine, which fits ellipses to an image using an iterative method described by Jedrzejewski [29]. In order to mask out foreground stars, SEXTRACTOR [30] was used. An inclination correction was then applied to the surface brightness profile $[31,32]$ as follows:

$$
\mu_{i}=\mu-2.5 C \log \left(\frac{a}{b}\right),
$$

where $\mu_{i}$ is the surface brightness when viewed at some inclination $i, \mu$ is the corrected surface brightness, $a$ is the major axis, $b$ is the minor axis, and $C$ is a factor dependent on whether the galaxy is optically thick or thin; if $C=1$ then the galaxy is optically thin; if $C=0$, then the galaxy is optically thick (e.g., [31, 32]). Graham [33] showed that $C=0.91$ is a good value to use for the near-infrared $K_{s}$ band. Adopting a simple reddening law, where extinction falls as the square of wavelength, it can be shown that a value of $C=0.97$ is appropriate at 3.6- $\mu \mathrm{m}[34]$, and we adopt this value here.

The resulting surface brighntess profile Figure 1 reaches a surface brightness of $\mu_{3.6} \sim 20.7 \mathrm{mag} \mathrm{arcsec}^{-2}$ at a radius of $\sim 13.2 \mathrm{kpc}$ (equivalent to $54.0 \mathrm{arcmin}$ ). From this surface brightness profile, we perform a one-dimensional bulgedisk decomposition, which employs the Sérsic model for 
Table 1: M33 Observational data. The Hubble type is from de Vaucouleurs et al. [17]. The distance in kpc is taken from Magrini et al. [21].

\begin{tabular}{lc}
\hline Parameter & Measurement \\
\hline Hubble type & SA $(\mathrm{s}) \mathrm{cd}$ \\
Distance $(\mathrm{kpc})$ & 840 \\
Position angle of major axis $\left(^{\circ}\right)$ & 23 \\
Bulge effective radius, $R_{e}(\operatorname{arcmin})$ & $1.60 \pm 0.11$ \\
Bulge effective radius, $R_{e}(\mathrm{kpc})$ & $0.39 \pm 0.03$ \\
Bulge surface brightness at the effective & $19.57 \pm 0.98$ \\
radius, $\mu_{e}\left(3.6 \mu \mathrm{m}-\right.$ mag arcsec $\left.^{-2}\right)$ & 1.0 \\
Bulge Sérsic index, $n$ & $18.08 \pm 1.02$ \\
Disk central surface brightness, & $6.95 \pm 0.49$ \\
$\mu_{0}(3.6 \mu$ m-mag arcsec & \\
Disk scalelength, $h(\operatorname{arcmin})$ & $1.70 \pm 0.12$ \\
Disk scalelength, $h(\mathrm{kpc})$ & $(3.16 \pm 0.30) \times 10^{9}$ \\
Disk luminosity, $L_{\text {disk }}\left(L_{\odot}\right)$ & 0.03 \\
Bulge-to-disk ratio, $B / D$ & \\
\hline
\end{tabular}

the bulge component and an exponential law for the disk component (e.g., [32, 35-39]; see [40] for a review). The Sérsic $[41,42] R^{1 / n}$ model is most commonly expressed as a surface brightness profile such that

$$
\mu(R)=\mu_{e} \exp \left(-b_{n}\left[\left(\frac{R}{R_{e}}\right)^{1 / n}-1\right]\right),
$$

where $\mu_{e}$ is the surface brightness at the effective radius $R_{e}$ that encloses half of the total light from the model $[43,44]$. The constant $b_{n}$ is defined in terms of the parameter $n$, which described the overall shape of the light profile. When $n=4$, the Sérsic model is equivalent to a de Vaucouleurs $[45,46]$ $R^{1 / 4}$ model, and when $n=1$, it is equivalent to an exponential model. The parameter $b_{n}$ has been approximated by $b_{n}=$ $1.9992 n-0.3271$, for $0.5<n<10[47,48]$. The exponential model for the disk surface brightness profile can be written as follows:

$$
\mu(R)=\mu_{0} \exp \left(-\frac{R}{h}\right)
$$

where $\mu_{0}$ is the disk central surface brightness and $h$ is the disk exponential scalelength. The results of our surface brightness fitting are summarized in Table 1.

We now assign masses to the disk and bulge of M33. The stellar mass-to-light ratio in the $K_{s}$ band is a well-calibrated quantity [49] which depends on $B-R$ color. Seigar et al. [34] extended this to a 3.6- $\mu \mathrm{m}$ image of M31 using the population synthesis codes of Bruzual and Charlot [50] and Maraston [51]. Using their results, we find a central mass-to-light ratio of $M / L_{3.6} \simeq 1.25 \pm 0.10$ with a gradient of $-0.014 \mathrm{kpc}^{-1}$. This results in a disk mass of $M_{\text {disk }}=(3.81 \pm 0.47) \times 10^{9} \mathrm{M}_{\odot}$ and a bulge mass of $M_{\text {bulge }}=(1.14 \pm 0.14) \times 10^{8} M_{\odot}$ for M33.

A concern in using the $3.6-\mu \mathrm{m}$ Spitzer waveband to determine the underlying stellar mass is the effect of emission from hot dust in this waveband although this is probably only important in or near HII regions. In order to place some constraint on this, we have chosen to explore the emission from dust in the near-infrared $K$ band at $2.2 \mu \mathrm{m}$. Using nearinfrared spectroscopy at $2.2 \mu \mathrm{m}$, it has been shown that hot dust can account for up to 30 per cent of the continuum light observed at this wavelength in areas of active star formation, that is, spiral arms [52]. When averaged over the entire disk of a galaxy, this reduces to a 2 percent effect if one assumes that spiral arms can be up to $12^{\circ}$ in width. At $3.6 \mu \mathrm{m}$, this would, therefore, result in 3 percent of emitted light from dust.

Another concern for the 3.6- $\mu \mathrm{m}$ waveband would be the contribution from the polycyclic aromatic hydrocarbon (PAH) emission feature at $3.3 \mu \mathrm{m}$. However, an infrared space observatory (ISO) spectroscopic survey of actively starforming galaxies by Helou et al. [53] found that the 3.3- $\mu \mathrm{m}$ feature was very weak when they analysed the average $2.5-$ $11.6-\mu \mathrm{m}$ spectrum of 45 galaxies. The contribution of the $\mathrm{PAH}$ feature to the 3.6- $\mu \mathrm{m}$ Spitzer waveband is therefore, not a major concern.

One other important contribution to the baryonic mass of M33 is the gas mass. Corbelli and Salucci [16] have shown that beyond a radius of $10 \mathrm{kpc}$, the gas contributes about the same to the rotation curve as the stars. Since the best current estimate of the gas distribution comes from Corbelli and Salucci [16], we have chosen to adopt their model for the distribution of gas mass in M33.

3.2. The Dark Halo Contribution. A range of allowed dark matter halo masses and density profiles is now explored, using two models for dark matter halo density profiles, the pseudoisothermal model (e.g., [9], see [54]), and the Navarro, Frenk, and White ([3]; hereafter NFW) profile. A pseudoisothermal density profile is given by

$$
\rho(R)=\rho_{0} \frac{R_{c}^{2}}{R_{c}^{2}+R^{2}},
$$

which in terms of rotational velocity becomes

$$
V_{c}^{2}(R)=V_{c}^{2}(\infty)\left(1-\frac{R_{c}}{R} \tan ^{-1} \frac{R}{R_{c}}\right)
$$

where $R_{c}$ is the core radius and $\rho_{0}=V_{c}^{2}(\infty) / 4 \pi G R_{c}^{2}$. The NFW profile is given by

$$
\rho(R)=\frac{\delta_{c} \rho_{c}^{0}}{\left(R / R_{s}\right)\left(1+R / R_{s}\right)^{2}},
$$

where $R_{s}$ is a characteristic "inner" radius, $\rho_{c}^{0}$ is the present critical density, and $\delta_{c}$ is a characteristic overdensity. This overdensity is defined as

$$
\delta_{c}=\frac{100 c_{\mathrm{vir}}^{3}}{3}
$$

where $c_{\mathrm{vir}}=R_{\mathrm{vir}} / R_{\mathrm{s}}$ is the concentration parameter and

$$
g\left(c_{\mathrm{vir}}\right)=\frac{1}{\ln \left(1+c_{\mathrm{vir}}\right)-c_{\mathrm{vir}} /\left(1+c_{\mathrm{vir}}\right)} .
$$




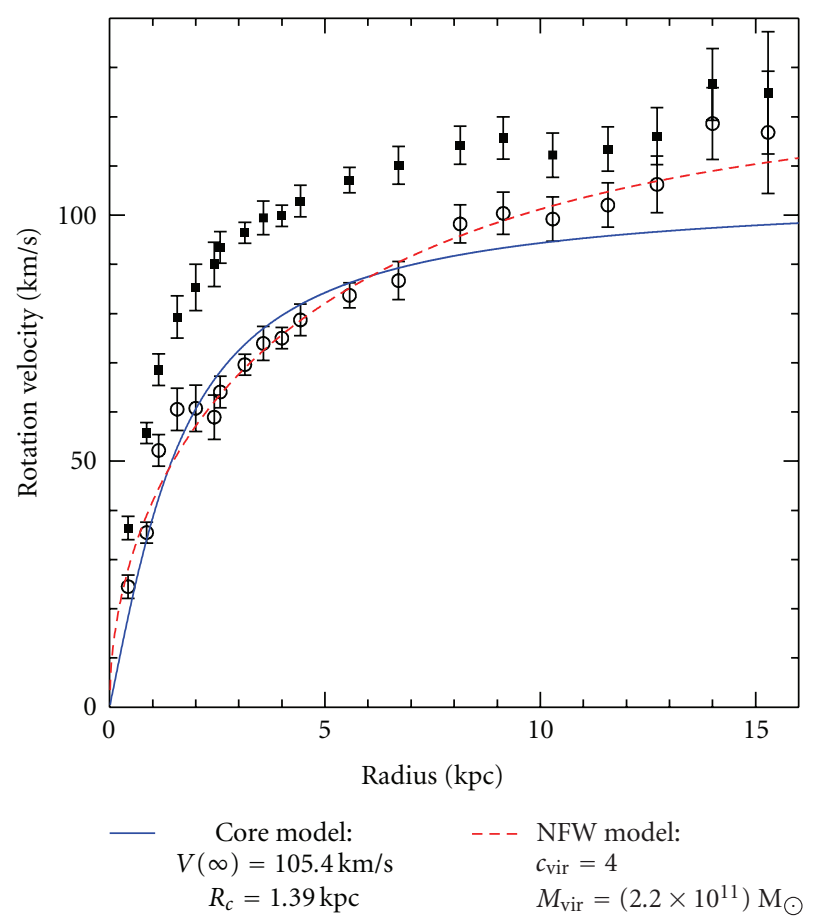

Figure 2: The HI rotation curve from Corbelli and Salucci [16] modeled using a pseudoisothermal model (core model; blue solid line) and an NFW model (red dotted line). The squares represent the total rotation velocities, and the circles represent the contribution of the dark matter to the rotation velocities (after subtraction of the stellar and gas mass).

The circular velocity associated with this density is given by Battaglia et al. [55] and is

$$
V_{c}^{2}=\frac{V_{\mathrm{vir}}^{2} g\left(c_{\mathrm{vir}}\right)}{s}\left[\ln \left(1+c_{\mathrm{vir}} s\right)-\frac{c_{\mathrm{vir}} s}{1+c_{\mathrm{vir}} s}\right],
$$

where $V_{\text {vir }}$ is the circular velocity at the virial radius $R_{\text {vir }}$ and $s=R / R_{\mathrm{vir}}$. This NFW profile is a two-parameter function and completely specified by choosing two independent parameters, for example, the virial mass $M_{\mathrm{vir}}$ (or virial radius $R_{\mathrm{vir}}$ ) and concentration $c_{\mathrm{vir}}=R_{\mathrm{vir}} / R_{s}$ (see [56] for a discussion). Similarly, given a virial mass $M_{\text {vir }}$ and the dark matter circular velocity at any radius, the halo concentration $c_{\text {vir }}$ is completely determined.

We now proceed by finding the best-fitting NFW and pseudoisothermal (or constant density core) dark matter halo density profiles that describe the complete HI rotation curve of M33 as observed by Crobelli and Salucci [16]. The result of this is shown in Figure 2. The pseudoisothermal fit is shown as the solid blue line, with best-fitting parameters of $V(\infty)=105.4 \pm 6.1 \mathrm{~km} \mathrm{~s}^{-1}$ and $R_{c}=1.39 \pm 0.04 \mathrm{kpc}$, and a reduced $-\chi^{2}$ value of $\chi^{2} / \mu=3.19$, where $\mu$ is the degrees of freedom. The NFW fit is shown as a dotted red line, with best-fitting parameters $c_{\text {vir }}=4.0 \pm 1.0$ and $M_{\text {vir }}=(2.2 \pm$ $0.1) \times 10^{11} \mathrm{M}_{\odot}$, with a reduced $-\chi^{2}$ value of $\chi^{2} / \mu=1$.18. As can be seen from Figure 2, the pseudoisothermal model (or core model in the figure) underestimates the rotation velocities beyond $\sim 7 \mathrm{kpc}$. However, the NFW fit more closely recreates
TABLE 2: M33 rotation curve modeling results, showing the bestfitting NFW and pseudoisothermal models.

\begin{tabular}{lc}
\hline Parameter & NFW model \\
\hline$M_{\text {vir }}$ & $(2.2 \pm 0.1) \times 10^{11} \mathrm{M}_{\odot}$ \\
$c_{\text {vir }}$ & $4.0 \pm 1.0$ \\
$\chi^{2} / \mu$ & 1.18 \\
\hline Parameter & Core model \\
\hline$R_{c}$ & $1.39 \pm 0.04 \mathrm{kpc}^{-1}$ \\
$V(\infty)$ & $105.4 \pm 6.1 \mathrm{~km} \mathrm{~s}^{-1}$ \\
$\chi^{2} / \mu$ & 3.19 \\
\hline
\end{tabular}

the observed data. This is also clear from the values of reduced $-\chi^{2}$. We therefore, conclude that the NFW model best represents these data, and this is consistent with the results of Corbelli and Salucci [16]. This is somewhat surprising for a late type, bulgeless galaxy like M33, since these late-type galaxies are often shown to have constant density cores (e.g., $[10,11])$.

Table 2 lists the best-fit parameters of the best-fit NSF and pseudoisothermal models based upon direct fitting to the HI rotation curve data.

It is probably worthwhile noting that our best-fitting NFW model yields a concentration parameter, $c_{\text {vir }}=$ $4.0 \pm 1.0$. This is somewhat lower than the concentration parameter of $c_{\text {vir }}=5.6$ reported by Corbelli and Salucci [16]. Furthermore, we derive a virial mass of $M_{\text {vir }}=(2.2 \pm 0.1) \times$ $10^{11} \mathrm{M}_{\odot}$, which is significantly lower than the virial mass of $M_{\text {vir }}=7.4 \times 10^{11} \mathrm{M}_{\odot}$ found by [16]. Here, we discuss some reasons that could account for these apparent differences. Since we use the same gas distribution as in Crobelli and Salucci [16], the only difference can come from the stellar mass component. The main difference between our stellar mass component, and that of Crobelli and Salucci [16], is that ours is determined from a Spitzer 3.6- $\mu \mathrm{m}$ observed in 2007, and that of Crobelli and Salucci [16] is determined from a $K$ band image reported by [57]. The $K$ band image from 1994 was taken when near-infrared arrays were really in their infancy, and so, it is probably more important to rely on the more modern datasets when possible. Furthermore, Crobelli and Salucci [16] assume a distance to M33 of $0.7 \mathrm{Mpc}$, whereas we use the more accurate measurement of $0.84 \mathrm{Mpc}$ from Magrini et al. [21]. As a result of this underestimatation in the distance to M33, Crobelli and Salucci [16] has underestimated the size of the visible galaxy by a factor of $\sim 17$ percent and this in turn has probably affected the total mass of M33 that they derive. Taking into account the different distances to M33, the disk scalelength of $h=1.2 \mathrm{kpc}$ used by Crobelli and Salucci [16] would become $h=1.4 \mathrm{kpc}$ if they had used the more accurate distance of $0.84 \mathrm{Mpc}$. This is still lower than the scalelngth of $h=1.7 \mathrm{kpc}$ that we report here. In converting this light distribution into stellar mass, we have then used a combination of the stellar mass-to-light ratios from Bell et al. [49] and the population synthesis codes from Maraston [51]. These papers provide the best estimates currently available for determining the stellar mass-to-light ratios, and they were not available to 
Corbelli and Salucci when they performed their analysis. One final difference between our results and those of Crobelli and Salucci [16] is that we include the bulge mass although considering the bulge-to-disk ratio of $B / D=0.03$ this is unlikely to have a significant effect on the mass models. As a result, we conclude that the differences between our results and those of Crobelli and Salucci [16] are caused by the different treatment of the disk starlight, updated stellar massto-light ratios, and more recent data.

Finally, it should be noted that Corbelli and Walterbos [58] revealed that M33 has a weak central bar. This could potentially have the affect of inducing noncircular motions in the central regions, that is, within $1 \mathrm{kpc}$. However, Kuzio de Naray and Kaufmann [59] have shown that, even in the case of barred galaxies, it is difficult to confuse an NFW dark matter halo profile with that of a pseudoisothermal profile. In other words, our result that M33 is best described by an NFW profile still holds, and given that the potential of the stellar bar is weak, the concentration is unlikely to change significantly.

In the following discussion, we use the NFW concentration parameter to reveal some interesting relationships.

\section{Discussion}

Seigar et al. [27, 28, 60] have demonstrated that a relationship exists between spiral arm pitch angle and rotation curve shear Rotation curve shear is defined as:

$$
S=\frac{A}{\omega}=\frac{1}{2}\left(1-\frac{R}{V} \frac{d V}{d R}\right),
$$

where $A$ is the first Oort constant, $\omega$ is the angular velocity, and $V$ is the velocity measured at radius $R$. Using this equation it is possible to determine the shear from a rotation curve. We have performed such an analysis on the HI rotation curve for M33 and found a value for its shear of $S=0.46 \pm 0.01$. We have also measured the spiral arm pitch angle for $\mathrm{M} 33$, which turns out to be $P=42 .^{\circ} 2 \pm 0 .{ }^{\circ} 3$ [61]. This pitch angle is in good agreement with previous measurements $[62,63]$. Figure 3 shows the relationship between spiral arm pitch angle and rotation curve shear. One can easily see that the pitch angle and shear values for M33 are consistent with the overall relationship.

Given the spiral arm pitch angles of a number of other galaxies, we can also now compare this quantity with the NFW concentration parameters for the galaxies listed in Table 3. Figure 4 shows a plot of NFW concentration as a function of spiral arm pitch angle in degrees. This plot may only be for 5 galaxies, but a relatively strong correlation appears to exist between these two quantities. Indeed, Pearson's linear correlation coefficient is 0.95 for this plot although the significance at which the null hypothesis of zero correlation is disproved in onlt 54 percent, probably due to low number statistics. Nevertheless, an interesting correlation seems to exist between spiral arm morphology and dark matter concentration, and this could be further studied by targeting more galaxies in an observational campaign. Indeed, these data seem consistent with the suggestion that pitch angle and mass concentration are related $[27,28]$.

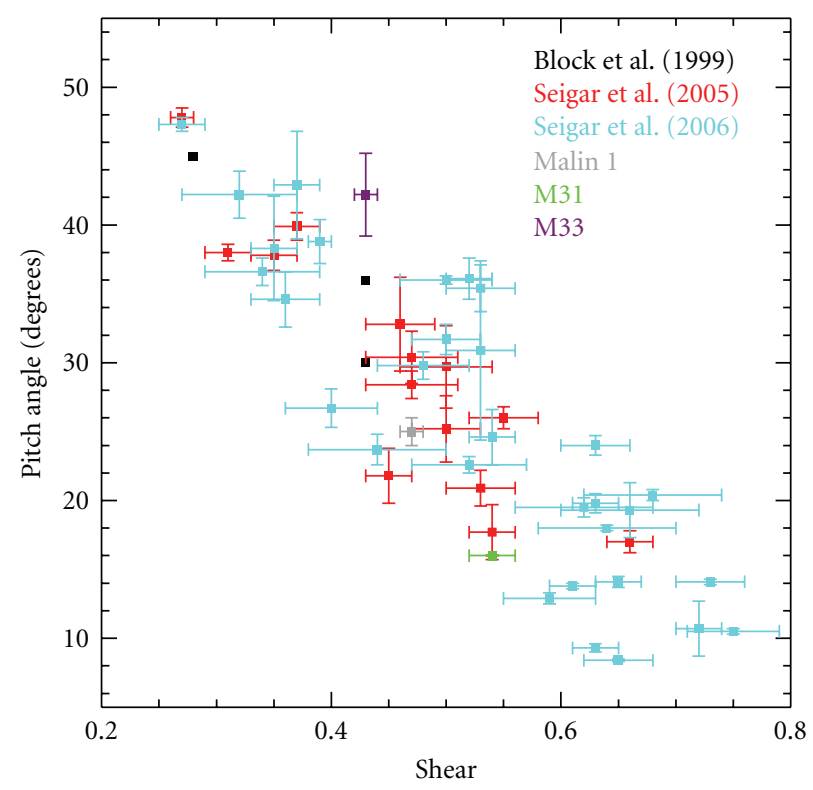

FIGURE 3: Spiral arm pitch angle versus rotation curve shear, showing a strong correlation. The solid squares represent galaxies with data measured by Block et al. [26], the red squares are galaxies from Seigar et al. [27], the blue squares are galaxies from Seigar et al. [28], the cyan square is for Malin 1 [64], the green square is for M31 [34], and the magenta square represents the data for M33 (this paper).

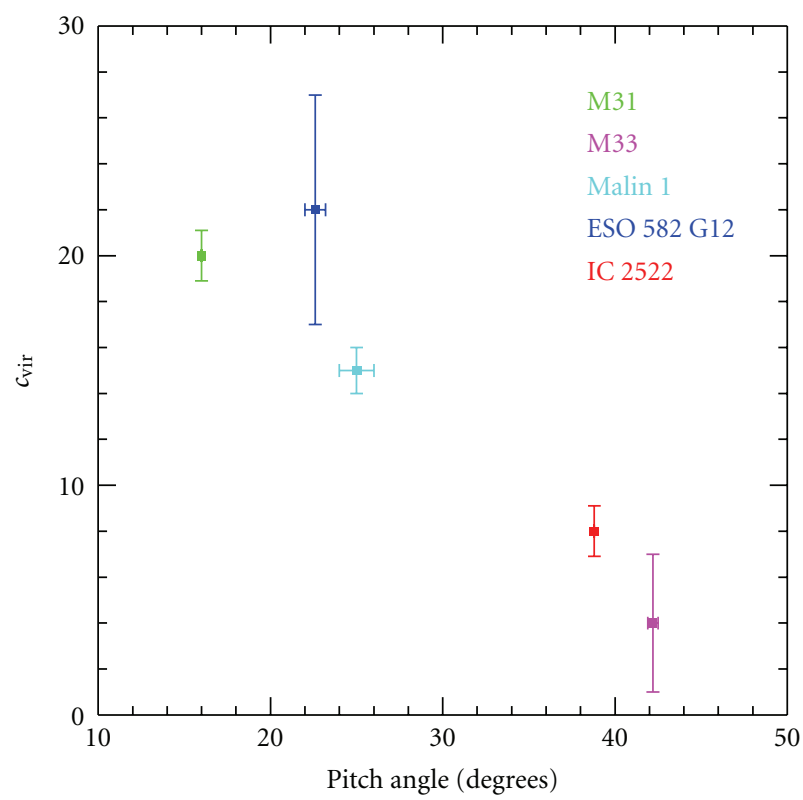

FIGURE 4: NFW concentration parameter versus spiral arm pitch angle showing a correlation. The green point represents data for M31, the cyan point for Malin 1, the red point for IC2522, the blue point for ESO582G12 and the magenta point shows the data for M33.

Finally Figure 5 shows a plot of supermassive black hole mass as a function of NFW concentration parameter. 
TABLe 3: Spiral arm pitch angles, NFW concentration parameters, and central supermassive black hole mass for 5 galaxies. For the Malin 1 the spiral arm pitch angle is taken from Moore and Parker [65]. For M31, the pitch angle is the average of values taken from Arp [66] and Braun [67]. The NFW concentration value is taken from (1) Seigar [64], (2) Klypin et al. [68], (3) Seigar et al. [34], and (4) Seigar et al. [28]. The black hole mass estimates are taken from (5) Ghez et al. [69], (6) Bender et al. [54], and (7) Gebhardt et al. [18].

\begin{tabular}{|c|c|c|c|c|c|}
\hline Galaxy name & Spiral arm pitch angle (degrees) & $c_{\mathrm{vir}}$ & Source & $M_{\mathrm{BH}}\left(\mathrm{M}_{\odot}\right)$ & Source \\
\hline Malin 1 & $25.0 \pm 1.0$ & $8.0 \pm 1.0$ & $(1)$ & - & - \\
\hline Milky Way & - & 12.0 & (2) & $(3.7 \pm 0.2) \times 10^{6}$ & (5) \\
\hline M31 & $7.1 \pm 0.4$ & $20.0 \pm 1.1$ & (3) & $(1.7 \pm 0.6) \times 10^{8}$ & (6) \\
\hline M33 & $42.2 \pm 3.0$ & 4.0 & - & $<1500$ & (7) \\
\hline IC2522 & $38.8 \pm 1.6$ & $8.0 \pm 1.0$ & $(4)$ & - & - \\
\hline ESO582G12 & $22.6 \pm 0.6$ & $22.0 \pm 5.0$ & (4) & - & - \\
\hline
\end{tabular}

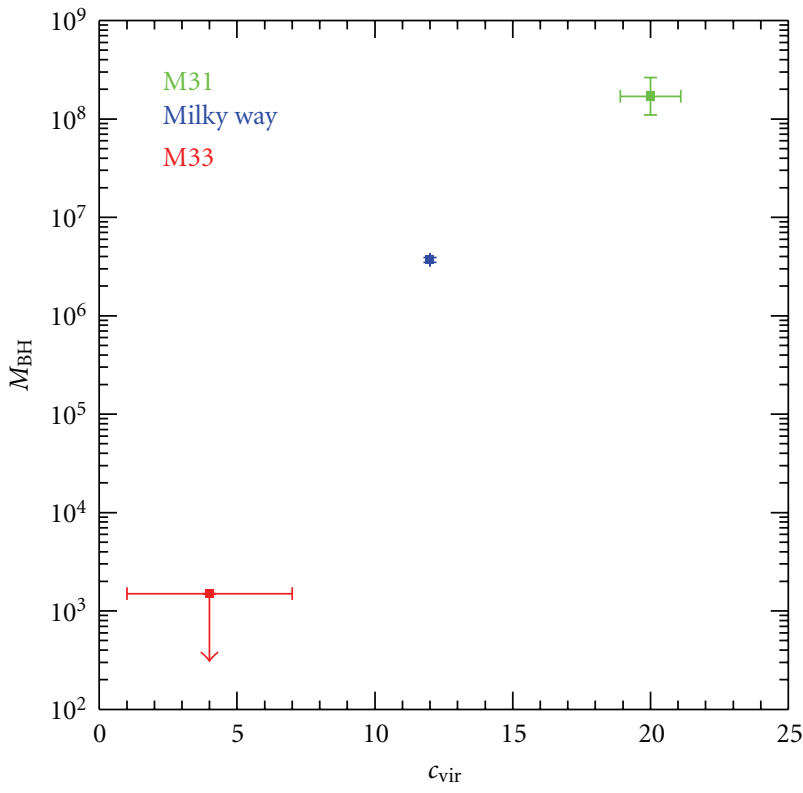

Figure 5: Central supermassive black hole mass versus NFW concentration parameter, showing a correlation. The green point represents data for M31, the blue point for the Milky Way, and the red point shows the data for M33.

Unfortunately, here, we only have data for three galaxies. Nevertheless, a hint of a correlation is starting to show, and seeing that such a correlation has been suggested by Seigar et al. [61], as well as Satyapal et al. [70] and Booth and Schaye [71], this plot is somewhat intriguing. This hint of a correlation should, of course, be expanded on by studying more galaxies along the Hubble sequence from type Sa to Sd.

\section{Summary}

We have shown that the HI rotation curve of M33 can be best modeled with a dark matter halo that follows a NFW profile with low NFW concentration of $c_{\text {vir }}=4.0$. Using the NFW concentration parameter from this fit, we find that interesting correlations between (1) spiral arm pitch angle and NFW concentration and (2) central supermassive black hole mass and NFW concentration start to appear. Although the second correlation is only for three galaxies, on the surface, it appears to be in disagreement with the argument made by Kormendy and Bender [72] that the dark matter halos of galaxies have no affect on the masses of supermassive black holes found in their centers. These correlations are very intriguing and our results warrant further investigation, as we have been limited to data that was available for just a few galaxies.

\section{Acknowledgments}

This research has made use of the NASA/IPAC Infrared Science Archive, which is operated by the Jet Propulsion Laboratory, California Institute of Technology, under contract with the National Aeronautics and Space Administration. The author wishes to thank the reviewers who helped to improve the content of this paper.

\section{References}

[1] G. R. Blumenthal, S. M Faber, R. Flores, and J. R. Primack, "Contraction of dark matter galactic halos due to baryonic infall," The Astrophysical Journal, vol. 301, pp. 27-34, 1986.

[2] V. Springel, S. D. M. White, A. Jenkins et al., "Simulations of the formation, evolution and clustering of galaxies and quasars," Nature, vol. 435, no. 7042, pp. 629-636, 2005.

[3] J. F. Navarro, C. S. Frenk, and S. D. M. White, "A universal density profile from hierarchical clustering," The Astrophysical Journal, vol. 490, no. 2, pp. 493-508, 1997.

[4] J. F. Navarro, E. Hayashi, C. Power et al., "The inner structure of $\Lambda \mathrm{CDM}$ haloes-III. Universality and asymptotic slopes," Monthly Notices of the Royal Astronomical Society, vol. 349, no. 3, pp. 1039-1051, 2004.

[5] J. Diemand, M. Zemp, B. Moore, J. Stadel, and C. M. Carollo, "Cusps in cold dark matter haloes," Monthly Notices of the Royal Astronomical Society, vol. 264, no. 2, pp. 665-673, 2005.

[6] R. A. Swaters, B. F. Madore, F. C. Van Den Bosch, and M. Balcells, "The central mass distribution in dwarf and low surface brightness galaxies," The Astrophysical Journal, vol. 583, no. 2, pp. 732-751, 2003.

[7] G. Gentile, P. Salucci, U. Klein, D. Vergani, and P. Kalberla, "The cored distribution of dark matter in spiral galaxies," Monthly Notices of the Royal Astronomical Society, vol. 351, no. 3, pp. 903-922, 2004.

[8] G. Gentile, A. Burkert, P. Salucci, U. Klein, and F. Walter, "The dwarf galaxy DDO 47 as a dark matter laboratory: testing cusps hiding in triaxial halos," The Astrophysical Journal, vol. 634, no. 2, pp. L145-L148, 2005. 
[9] J. D. Simon, A. D. Bolatto, A. Leroy, L. Blitz, and E. L. Gates, "High-resolution measurements of the halos of four dark matter-dominated galaxies: deviations from a universal density profile," The Astrophysical Journal, vol. 621, no. 2, pp. 757-776, 2005.

[10] R. K. De Naray, S. S. McGaugh, W. J. G. De Blok, and A. Bosma, "High-resolution optical velocity fields of 11 low surface brightness galaxies," The Astrophysical Journal, Supplement Series, vol. 165, no. 2, pp. 461-479, 2006.

[11] R. K. De Naray, S. S. McGaugh, and W. J. G. De Blok, "Mass models for low surface brightness galaxies with highresolution optical velocity fields," The Astrophysical Journal, vol. 676, no. 2, pp. 920-943, 2008.

[12] F. Shankar, A. Lapi, P. Salucci, G. De Zotti, and L. Danese, "New relationships between galaxy properties and host halo mass, and the role of feedbacks in galaxy formation," The Astrophysical Journal, vol. 643, no. 1, pp. 14-25, 2006.

[13] M. Spano, M. Marcelin, P. Amram, C. Carignan, B. Epinat, and O. Hernandez, "GHASP: an $\mathrm{H} \alpha$ kinematic survey of spiral and irregular galaxies-V. Dark matter distribution in 36 nearby spiral galaxies," Monthly Notices of the Royal Astronomical Society, vol. 383, no. 1, pp. 297-316, 2008.

[14] A. R. Zentner and J. S. Bullock, "Inflation, cold dark matter, and the central density problem," Physical Review D, vol. 66, no. 4, Article ID 043003, 12 pages, 2002.

[15] R. H. Wechsler, J. S. Bullock, J. R. Primack, A. V. Kravtsov, and A. Dekel, "Concentrations of dark halos from their assembly histories," The Astrophysical Journal, vol. 568, no. 1, pp. 52-70, 2002.

[16] E. Corbelli and P. Salucci, "The extended rotation curve and the dark matter halo of M33," Monthly Notices of the Royal Astronomical Society, vol. 311, no. 2, pp. 441-447, 2000.

[17] G. de Vaucouleurs, A. de Vaucouleurs, H. G. Corwin Jr., R. J. Buta, G. Paturel, and R. Fouqué, The Third Reference Catalog of Bright Galaxies, Springer, New York, NY, USA, 1991.

[18] K. Gebhardt, T. R. Lauer, J. Kormendy et al., "M33: a galaxy with no supermassive black hole," The Astronomical Journal, vol. 122, pp. 2469-2476, 2001.

[19] J. Magorrian, S. Tremaine, D. Richstone et al., "The demography of massive dark objects in galaxy centers," The Astronomical Journal, vol. 115, no. 6, pp. 2285-2305, 1998.

[20] N. Häring and H.-W. Rix, "On the black hole mass-bulge mass relation,” The Astrophysical Journal, vol. 604, no. 2, p. L89, 2004.

[21] L. Magrini, E. Corbelli, and D. Galli, "The building up of the disk galaxy M 33 and the evolution of the metallicity gradient," Astronomy and Astrophysics, vol. 470, no. 3, pp. 843-855, 2007.

[22] G. Paturel, C. Petit, PH. Prugniel et al., "HYPERLEDA. I: identification and designation of galaxies," Astronomy and Astrophysics, vol. 412, no. 1, pp. 45-55, 2003.

[23] M. F. S. Schröder, M. G. Pastoriza, S. O. Kepler, and I. Puerari, "The distribution of light in the spiral galaxy NGC 7412," Search Results Astronomy and Astrophysics Supplement Series, vol. 108, pp. 41-54, 1994.

[24] S. Considére and E. Athanassoula, "Analysis of spiral components in 16 galaxies," Astronomy and Astrophysics Supplement Series, vol. 76, no. 3, pp. 365-404, 1988.

[25] C. Garcia-Gomez and E. Athanassoula, "Analysis of the distribution of H II regions in external galaxies. II-analysis of the spiral structure," Astronomy and Astrophysics Supplement Series, vol. 100, no. 2, pp. 431-464, 1993.

[26] D. L. Block, I. Puerari, J. A. Frogel, P. B. Eskridge, A. Stockton, and B. Fuchs, "Cosmic masks still dance," Astrophysics and Space Science, vol. 269-270, no. 1-4, pp. 5-29, 1999.
[27] M. S. Seigar, D. L. Block, I. Puerari, N. E. Chorney, and P. A. James, "Dust-penetrated arm classes: insights from rising and falling rotation curves," Monthly Notices of the Royal Astronomical Society, vol. 359, no. 3, pp. 1065-1076, 2005.

[28] M. S. Seigar, D. L. Block, I. Puerari, N. E. Chorney, and P. A. James, "Constraining dark matter halo profiles and galaxy formation models using spiral arm morphology. I. Method outline," The Astrophysical Journal, vol. 645, no. 2, pp. 1012 1023, 2006.

[29] R. I. Jedrzejewski, "CCD surface photometry of elliptical galaxies. I-observations, reduction and results," Monthly Notices of the Royal Astronomical Society, vol. 226, pp. 747-768, 1987.

[30] E. Bertin and S. Arnouts, "SExtractor: software for source extraction," Astronomy and Astrophysics Supplement Series, vol. 117, no. 2, pp. 393-404, 1996.

[31] R. S. de Jong, "Near-IR photometry of 86 galaxies. II," Astronomy and Astrophysics Supplement Series, vol. 118, p. 557, 1996.

[32] M. S. Seigar and P. A. James, "The structure of spiral galaxiesI. Near-infrared properties of bulges, discs and bars," Monthly Notices of the Royal Astronomical Society, vol. 299, no. 3, pp. 672-684, 1998.

[33] A. W. Graham, "A K-band central disc surface brightness correlation with scalelength for early-type disc galaxies, and the inclination correction," Monthly Notices of the Royal Astronomical Society, vol. 326, no. 2, pp. 543-552, 2001.

[34] M. S. Seigar, A. J. Barth, and J. S. Bullock, "A revised $\Lambda$ CDM mass model for the Andromeda Galaxy," Monthly Notices of the Royal Astronomical Society, vol. 389, no. 4, pp. 1911-1923, 2008.

[35] Y. C. Andredakis, R. F. Peletier, and M. Balcells, "The shape of the luminosity profiles of bulges of spiral galaxies," Search Results Monthly Notices of the Royal Astronomical Society, vol. 275, no. 3, p. 874, 1995.

[36] H. G. Khosroshahi, Y. Wadadekar, and A. Kembhavi, "Correlations among global photometric properties of disk galaxies," The Astrophysical Journal, vol. 533, no. 1, pp. 162-171, 2000.

[37] M. D'Onofrio, “2D modelling of the light distribution of earlytype galaxies in a volume-limited sample-II. Results for real galaxies," Monthly Notices of the Royal Astronomical Society, vol. 326, no. 4, pp. 1517-1532, 2001.

[38] A. W. Graham, "An investigation into the prominence of spiral galaxy bulges," The Astronomical Journal, vol. 121, no. 2, pp. 820-840, 2001.

[39] C. Möllenhoff and J. Heidt, "Surface photometry of spiral galaxies in NIR: structural parameters of disks and bulges," Astronomy and Astrophysics, vol. 368, no. 1, pp. 16-37, 2001.

[40] A. W. Graham and S. P. Driver, "A concise reference to (projected) sérsic $1 / \mathrm{n}$ quantities, including concentration, profile slopes, petrosian indices, and kron magnitudes," Publications of the Astronomical Society of Australia, vol. 22, no. 2, pp. 118127, 2005.

[41] J. L. Sérsic, "Influence of the atmospheric and instrumental dispersion on the brightness distribution in a galaxy," Boletin de la Asociacion Argentina de Astronomia, vol. 6, p. 41, 1963.

[42] J. L. Sérsic, "Atlas de Galaxies Australes. Observatorio Astronomico," Cordoba, 1968.

[43] L. Ciotti, "Stellar systems following the R exp 1/m luminosity law," Astronomy and Astrophysics, vol. 249, no. 1, pp. 99-106, 1989.

[44] N. Caon, M. Capaccioli, and M. D'Onofrio, "On the shape of the light profiles of early type galaxies," Monthly Notices of the Royal Astronomical Society, vol. 265, no. 4, p. 1013, 1993. 
[45] G. de Vaucouleurs, "Recherches sur ies nebuleuses extragalactiques," Annales d'Astrophysique, vol. 11, p. 247, 1948.

[46] G. de Vaucouleurs, "Classification and morphology of external galaxies," Handbuch der Physik, vol. 53, pp. 275-310, 1959.

[47] M. Capaccioli, "Photometry of early-type galaxies and the R exp 1/4 law," in The World of Galaxies, H. G. Corwin and L. Bottinelli, Eds., pp. 208-227, Springer, Berlin, Germany, 1989.

[48] P. H. Prugniel and F. Simien, "The fundamental plane of early-type galaxies: non-homology of the spatial structure," Astronomy and Astrophysics, vol. 321, no. 1, pp. 111-122, 1997.

[49] E. F. Bell, D. H. McIntosh, N. Katz, and M. D. Weinberg, "A first estimate of the baryonic mass function of galaxies," The Astrophysical Journal, vol. 585, no. 2, pp. L117-L120, 2003.

[50] G. Bruzual and S. Charlot, "Stellar population synthesis at the resolution of 2003," Monthly Notices of the Royal Astronomical Society, vol. 344, no. 4, pp. 1000-1028, 2003.

[51] C. Maraston, "Evolutionary population synthesis: models, analysis of the ingredients and application to high-z galaxies," Monthly Notices of the Royal Astronomical Society, vol. 362, no. 3, pp. 799-825, 2005.

[52] P. A. James and M. S. Seigar, "The nature of near-infrared emission from spiral galaxies," Astronomy and Astrophysics, vol. 350, no. 3, pp. 791-796, 1999.

[53] G. Helou, N. Y. Lu, M. W. Werner, S. Malhotra, and N. Silbermann, "The mid-infrared spectra of normal galaxies," The Astrophysical Journal, vol. 532, no. 1, pp. L21-L24, 2000.

[54] R. Bender, J. Kormendy, G. Bower et al., "HST STIS spectroscopy of the triple nucleus of M31: two nested disks in keplerian rotation around a supermassive black hole," The Astrophysical Journal, vol. 631, no. 1, pp. 280-300, 2005.

[55] G. Battaglia, A. Helmi, H. Morrison et al., "The radial velocity dispersion profile of the Galactic halo: constraining the density profile of the dark halo of the Milky Way," Monthly Notices of the Royal Astronomical Society, vol. 364, no. 2, pp. 433-442, 2005.

[56] J. S. Bullock, T. S. Kolatt, A. Dekel et al., "Profiles of dark haloes: evolution, scatter and environment," The Astrophysical Journal, vol. 555, no. 1, pp. 240-257, 2001.

[57] M. W. Regan and S. N. Vogel, "The near-infrared structure of M33,” The Astrophysical Journal, vol. 434, no. 2, pp. 536-545, 1994.

[58] E. Corbelli and R. A. M. Walterbos, "Bar imprints on the inner gas kinematics of M33," The American Astronomical Society, vol. 669, pp. 315-326, 2007.

[59] R. K De Naray and T. Kaufmann, "Recovering cores and cusps in dark matter haloes using mock velocity field observations," Monthly Notices of the Royal Astronomical Society. In press, http://arxiv.org/abs/1012.3471.

[60] M. S. Seigar, D. L. Block, and I. Puerari, "Dust penetrated arm classes-insights from rising and falling rotation curves"', in Penetrating Bars Through Masks of Cosmic Dust: The Hubble Tuning Fork Strikes a New Note, L. Block, I. Puerari, K. C. Freeman, R. Groess, and E. K. Block, Eds., Springer, Dordrecht, The Netherlands, 2004.

[61] M. S. Seigar, D. Kennefick, J. Kennefick, and C. H.S. Lacy, "Discovery of a relationship between spiral arm morphology and supermassive black hole mass in disk galaxies," The Astrophysical Journal, vol. 678, no. 2, pp. L93-L96, 2008.

[62] A. Sandage and R. M. Humphreys, "On the warped optical plane of M33," The Astrophysical Journal, vol. 236, pp. L1-L5, 1980.

[63] D. L. Block, K. C. Freeman, T. H. Jarrett et al., "Very luminous carbon stars in the outer disk of the Triangulum spiral galaxy," Astronomy and Astrophysics, vol. 425, no. 3, pp. L37-L40, 2004.
[64] M. S. Seigar, "A cosmologically motivated description of the dark matter halo profile for the low surface brightness galaxy, Malin 1," Publications of the Astronomical Society of the Pacific, vol. 120, no. 871, pp. 945-951, 2008.

[65] L. Moore and Q. A. Parker, "Malin 1: a deeper look," Publications of the Astronomical Society of Australia, vol. 23, no. 4, pp. 165-169, 2006.

[66] H. Arp, "A technique for faint photography applied to the radio source in fornax," The Astrophysical Journal, vol. 139, p. 1378, 1964.

[67] R. Braun, "The distribution and kinematics of neutral gas in M31," The Astrophysical Journal, vol. 372, no. 1, pp. 54-66, 1991.

[68] A. Klypin, H. Zhao, and R. S. Somerville, " $\Lambda$ CDM-based models for the Milky Way and M31. I. Dynamical models," The Astrophysical Journal, vol. 573, no. 2, pp. 597-613, 2002.

[69] A. M. Ghez, S. Salim, S. D. Hornstein et al., "Stellar orbits around the galactic center black hole," The Astrophysical Journal, vol. 620, no. 2, pp. 744-757, 2005.

[70] S. Satyapal, D. Vega, R. P. Dudik, N. P. Abel, and T. Heckman, "Spitzer uncovers active galactic nuclei missed by optical surveys in seven late-type galaxies," The Astrophysical Journal, vol. 677, no. 2, pp. 926-942, 2008.

[71] C. M. Booth and J. Schaye, "Dark matter haloes determine the masses of supermassive black holes," Monthly Notices of the Royal Astronomical Society, vol. 405, no. 1, pp. L1-L5, 2010.

[72] J. Kormendy and R. Bender, "Supermassive black holes do not correlate with dark matter haloes of galaxies," Nature, vol. 469, no. 7330, pp. 377-380, 2011. 

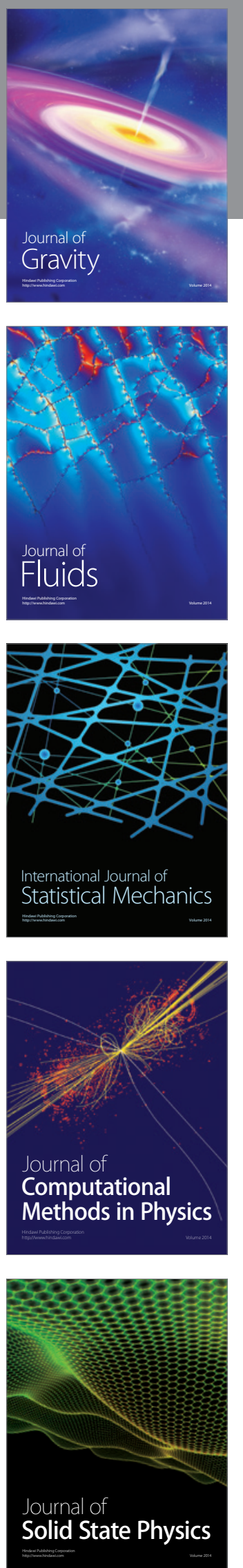

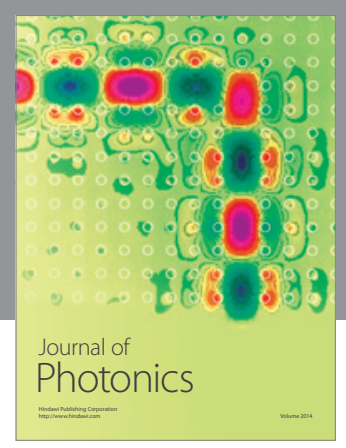

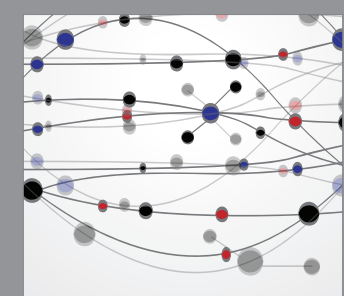

The Scientific World Journal
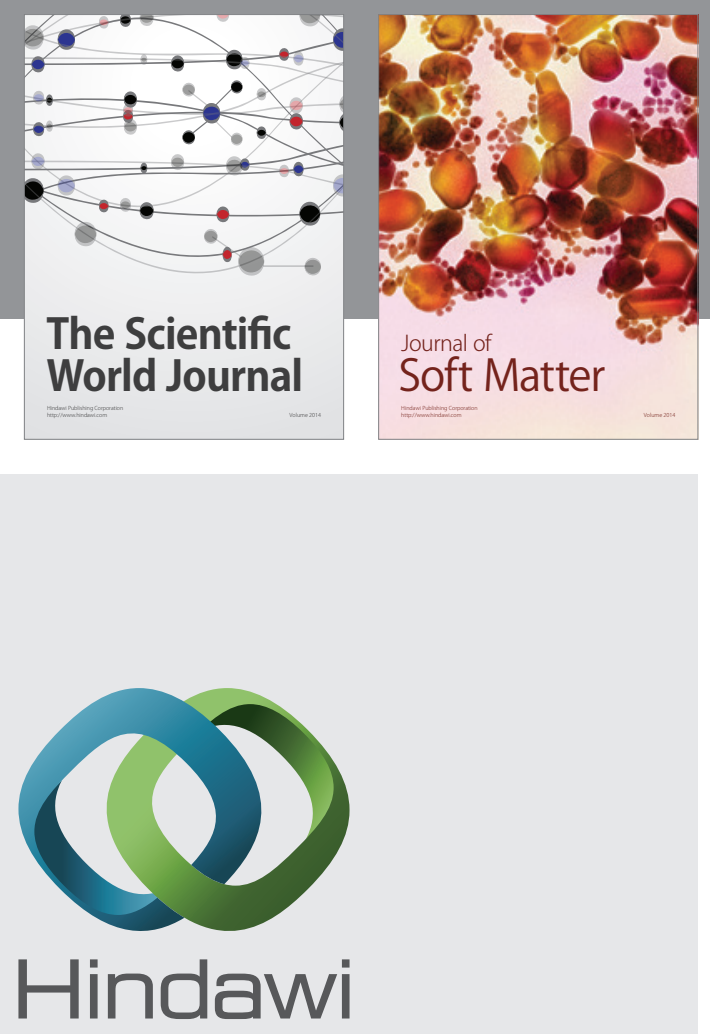

Submit your manuscripts at

http://www.hindawi.com
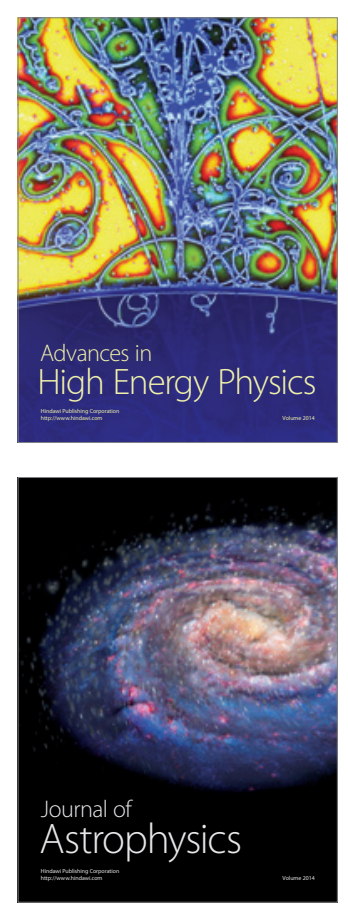
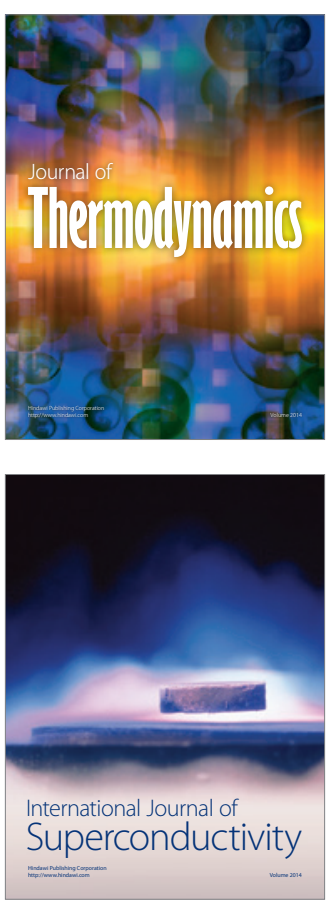
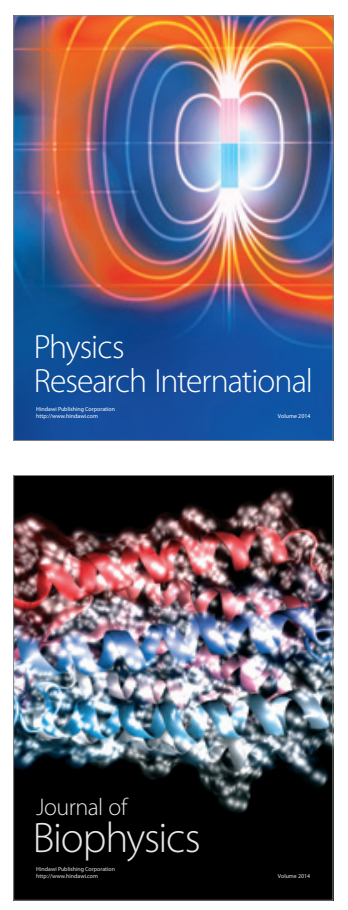
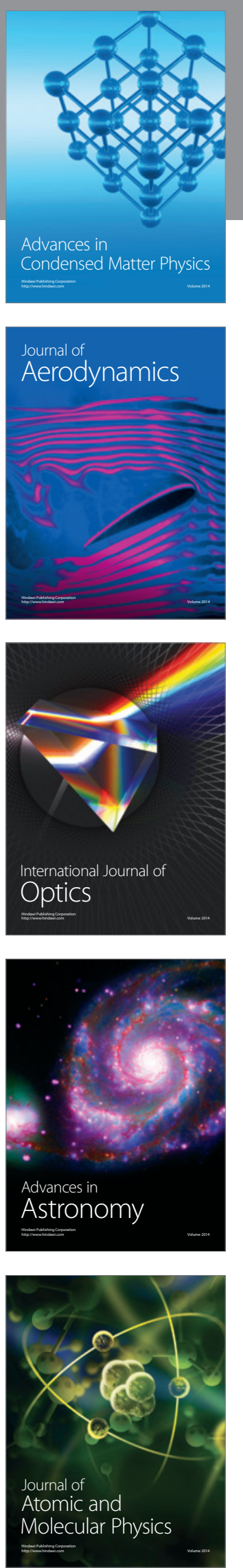